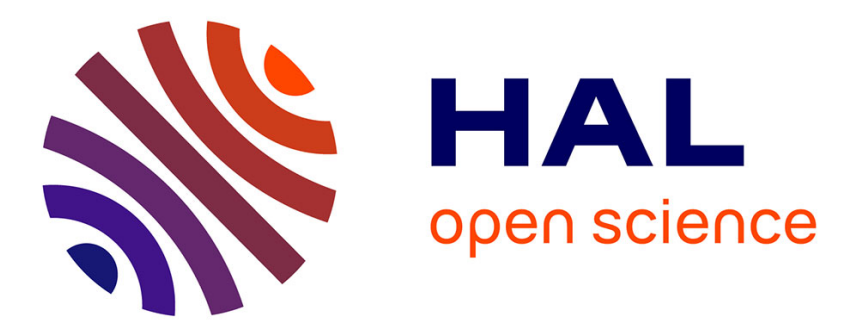

\title{
E.P.R. investigation of the incommensurate phase of ThBr4: Gd3+, through fourth order spin-crystal field interaction
}

\author{
S. Hubert, J. Emery, J.J. Rousseau, J.C. Fayet
}

\section{- To cite this version:}

S. Hubert, J. Emery, J.J. Rousseau, J.C. Fayet. E.P.R. investigation of the incommensurate phase of ThBr4: Gd3+, through fourth order spin-crystal field interaction. Journal de Physique Lettres, 1982, 43 (22), pp.815-821. 10.1051/jphyslet:019820043022081500 . jpa-00232130

HAL Id: jpa-00232130

https://hal.science/jpa-00232130

Submitted on 1 Jan 1982

HAL is a multi-disciplinary open access archive for the deposit and dissemination of scientific research documents, whether they are published or not. The documents may come from teaching and research institutions in France or abroad, or from public or private research centers.
L'archive ouverte pluridisciplinaire HAL, est destinée au dépôt et à la diffusion de documents scientifiques de niveau recherche, publiés ou non, émanant des établissements d'enseignement et de recherche français ou étrangers, des laboratoires publics ou privés. 
Classification

Physics Abstracts

$61.16 \mathrm{~N}-64.70-73.60 \mathrm{~F}$

\title{
E.P.R. investigation of the incommensurate phase of $\mathrm{ThBr}_{4}: \mathbf{G d}^{3+}$, through fourth order spin-crystal field interaction
}

\author{
S. Hubert (*), J. Emery (**), J. J. Rousseau (**) and J. C. Fayet (**) \\ (*) Laboratoire de Radiochimie, Institut de Physique Nucléaire, B.P. 1, 91406 Orsay, France \\ $\left(^{* *}\right)$ Laboratoire de Spectroscopie du Solide $\left({ }^{+}\right)$, Faculté des Sciences, 72017 Le Mans Cedex, France
}

(Reçu le 23 juin 1982, révisé le 23 septembre, accepté le ler octobre 1982)

\begin{abstract}
Résumé. - La forme de la raie de R.P.E. $\Delta M_{S}:\left|\frac{5}{2}\right| \rightarrow\left|\frac{7}{2}\right|$ pour le centre $\mathrm{Gd}^{3+}-$ n.n.n. $\mathrm{V}_{\left(\mathrm{Br}^{-}\right)}$peut être interprétée de façon cohérente en supposant une rotation modulée autour de c des liaisons $\mathrm{Th}-\mathrm{Br}$ comme composante essentielle du champ de déplacement dans la phase incommensurable. La sensibilité de la sonde $\mathrm{Gd}^{3+}$ provient de la grande valeur du paramètre du quatrième ordre $b_{4}^{4}$ de l'Hamiltonien de spin, liée à la configuration aplatie du tétrahèdre de $\mathrm{Br}^{-}$plus proches voisins. Nous donnons des résultats préliminaires et les grandes lignes de développements possibles de la présente étude.

Abstract. - The shape of the E.P.R. line $\Delta M_{S}:\left|\frac{5}{2}\right| \rightarrow\left|\frac{7}{2}\right|$ for the $\mathrm{Gd}^{3+}$-n.n.n. $\mathrm{V}_{\left(\mathrm{Br}^{-}\right)}$centre, can be consistently interpreted by assuming a modulated rotation around the c crystal axis of the $\mathrm{Th}-\mathrm{Br}$ bonds as an essential component of the displacement field in the incommensurate phase. The sensitivity of the local $\mathrm{Gd}^{3+}$ probe arises from the high value of the fourth order parameter $b_{4}^{4}$ linked to the flat configuration of the n.n. $\mathrm{Br}^{-}$tetrahedron. We report preliminary results and we outline possible developments of the present investigation.
\end{abstract}

1. Introduction. - Recently, Blinc et al. [1] have developed computer reconstructions of the particular shape of N.M.R., N.Q.R., E.P.R. lines in incommensurate phases, a simple model. We shall use their results for one dimensionally modulated systems.

Briefly, these authors assume that the local line shifts, $\Delta H$ or $\Delta \omega$, mirror the amplitude $u$ of the local atomic displacements through a polynomial law : $\Delta(H$ or $\omega)=a u+b u^{2}+\ldots$. This law depends on the particular conditions of the spin-crystal field interaction, the simplest case being a linear law. The different modulation regimes of $u$ (i.e. plane wave, broad or narrow soliton regimes) are connected to different integration constants of a Sine Gordon equation. The experimental N.M.R. line shapes of ${ }^{87} \mathrm{Rb}_{\text {in }} \mathrm{Rb}_{2} \mathrm{ZnCl}_{4}$ obey this model [1]. For E.P.R. lines in the same crystal doped with $\mathrm{Mn}^{2+}$ one may refer to $[2,3]$.

$\left({ }^{+}\right)$E.R.A. $n^{\circ} 682$. 
Recently a phase transition was observed by Raman scattering spectroscopy [4] and nuclear quadrupolar resonance [5] at $94 \mathrm{~K}$ for $\mathrm{ThBr}_{4}$ and $70 \mathrm{~K}$ for $\mathrm{ThCl}_{4}$. Single crystal neutron diffraction experiments revealed that the low-temperature structure is incommensurate with a modulation along the fourfold axis $[6,7]$ without any locking until $10 \mathrm{~K}$. At room temperature $\mathrm{ThBr}_{4}$ has a $\mathrm{D}_{4 \mathrm{~h}}^{19}$ tetragonal structure isostructural with $\mathrm{ThCl}_{4}$ and $\mathrm{UCl}_{4}[8]$. In this structure, the $\mathrm{Th}^{4+}$ ions are at a site of $D_{2 d}$ symmetry and a dopent ion substitutes into this site.

In this paper we have used $\mathrm{Gd}^{3+}$ substituted for $\mathrm{Th}^{4+}$ as an E.P.R. probe of the lattice properties. We shall emphasize that we have considered the short range fourth order spin-crystal field interaction as previously done for $\mathrm{RbCdF}_{3}: \mathrm{Gd}^{3+}$ below $T_{\mathrm{c}}[9]$. Therefore, the condition of validity of the model developed by Blinc, i.e. the short range character of the spin-probe, is fulfilled; indeed one may question this point when one uses, as usual, the quadrupolar spincrystal field interaction.

2. Experimental. - The single crystals doped with $0.05 \% \mathrm{of} \mathrm{Gd}^{3+}$ [10], grown by one of us (S. H.), were cooled by a regulated helium gas flow, with a temperature stability $\pm 0.05 \mathrm{~K}$ in the course of the experiment. We have neglected any temperature difference between the sensor and the sample. Nevertheless we assumed that this difference remained constant within a temperature range of few $\mathrm{K}$ around $T_{\mathrm{c}}$.

3. Tetragonal phase - In the doped sample, $\mathrm{Gd}^{3+}$ substitutes for $\mathrm{Th}^{4+}$ and charge compensation occurs through $\mathrm{Br}^{-}$vacancies. In freshly grown crystals, three types of paramagnetic centres were observed : $a D_{2 d}$ centre and two orthorhombic ones [11].

3.1 $\mathrm{D}_{2 \mathrm{~d}}$ CENTRE. - The $\mathrm{Gd}^{3+}$ ion with distant $\mathrm{Br}^{-}$vacancies gives rise to a $\mathrm{D}_{2 \mathrm{~d}}$ centre (Fig. 1). The corresponding spin Hamiltonian parameters have been previously determined : $b_{0}^{2}=460 ; b_{4}^{0}=10.0 ; b_{4}^{4}=123.0 ; b_{6}^{0}=1 ; b_{6}^{4}=3$, in $10^{-4} \mathrm{~cm}^{-1}[11]$ (note the large value of $b_{4}^{4}$ ). Using the superposition model [12] as a first approximation for the fourth order parameters we find :

$$
b_{4}^{0}=\frac{1}{2} \bar{b}_{4}\left(35 \cos ^{4} \alpha-30 \cos ^{2} \alpha+3\right) ; \quad b_{4}^{4}=\frac{35}{2} \bar{b}_{4} \sin ^{4} \alpha
$$

$\bar{b}_{4}$ is a parameter which characterizes the $\mathrm{Gd}^{3+}-\mathrm{n} . \mathrm{n}$. $\mathrm{Br}^{-}$bonds (n.n. for nearest neighbour),

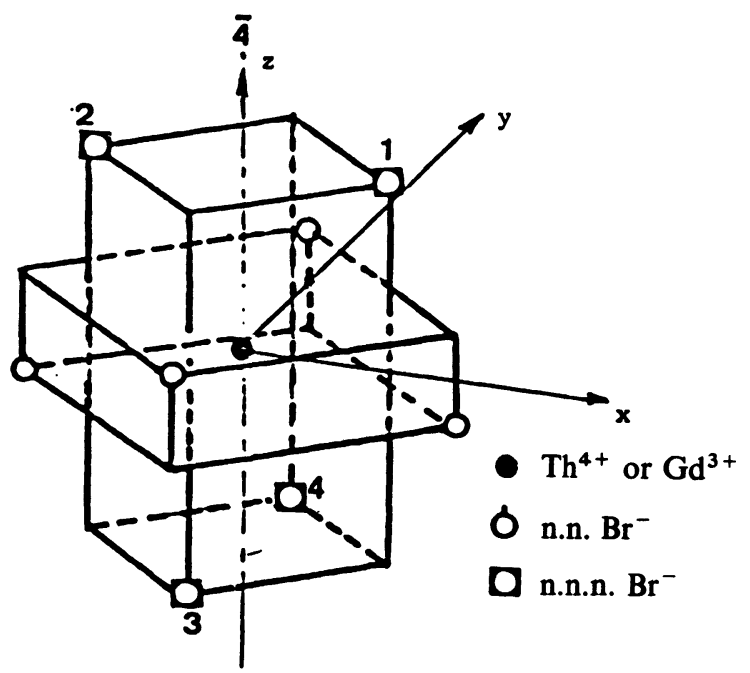

Fig. 1. - The $\mathrm{D}_{2 \mathrm{~d}}$ crystal site of $\mathrm{Th}^{4+}$ in $\mathrm{ThBr}_{4}$. The spin Hamiltonian axis $(x, y, z)$ of $\mathrm{Gd}^{3+}\left(\mathrm{D}_{2 \mathrm{~d}}\right)$ are along the crystal axes. 
and $\alpha=77^{\circ} 30^{\prime}$ is the angle of the bonds with the spin Hamiltonian axis, i.e. with the crystal axis $\mathrm{c}$. The flat configuration of the n.n. $\mathrm{Br}^{-}$tetrahedron $\left(\alpha \gg 55^{\circ}\right)$ accounts for the large value of $b_{4}^{4}$ and the calculated value of $b_{4}^{4} / b_{4}^{0} \simeq 20$ has a correct order of magnitude.

The consequence is a marked anisotropy of the high field line $\left|\frac{5}{2}\right| \rightarrow\left|\frac{7}{2}\right|$ when $H$ is rotated in the (001) plane. Indeed this anisotropy is given to first order, by $H(A, \theta)=H_{0}+A b_{4}^{4} \cos 4 \theta$ with $\theta=(\mathbf{a}, \mathbf{H})$ and $\mathbf{x}=\mathbf{a}$. The contribution of $b_{6}^{4}$ is neglected and $A$ is a constant.

Imagine a situation in which the local surroundings of the $\mathrm{Gd}^{3+}$ ion rotates by $\varepsilon$ around $\mathrm{c}$. The essential effect would be a tilt by $\varepsilon$ of the $\mathbf{x}$ spin Hamiltonian axis and a shift of the line by :

$$
\Delta H(\varepsilon) \sim b_{4}^{4}\{\cos [4(\theta+\varepsilon)]-\cos [4 \theta]\} .
$$

For $\theta=22^{\circ} 30^{\prime}$ the shift is maximum and linear in $\varepsilon$ up to $0\left(\varepsilon^{3}\right)$. For $\theta=0, \pi / 4$, the shift is minimum and quadratic in $\varepsilon$. For a physical illustration of this situation one may refer to the E.P.R. spectra of $\mathrm{RbCdF}_{3}: \mathrm{Gd}^{3+}-\mathrm{V}_{\left(\mathrm{Cd}^{2+}\right)}$ below $T_{\mathrm{c}}[9]$.

3.2 THE $\mathrm{Gd}^{3+}$-n.n.n. $\mathrm{V}_{\left(\mathrm{Br}^{-}\right)}$CENTRE. - The most intense lines in the sample under investigation were attributed to $\mathrm{Gd}^{3+}$ ions associated to n.n.n. $\mathrm{Br}^{-}$vacancies (n.n.n. for next n.n.). Indeed, the main features of these lines are not very different from the lines of the $D_{2 d}$ centre. This observation excludes charge compensation at a n.n. $\mathrm{Br}^{-}$site which would produce important modifications of the spin Hamiltonian parameters and axes. Nevertheless the symmetry of the centre is lowered from $D_{2 d}$ to $C_{s}$, and a $D_{2 d}$ site gives rise to four centres, depending on the location of the n.n.n. vacancy at sites $1,2,3$ or 4 (Fig. 1).

When $H$ rotates in the $(001)$ plane, these centres give rise to a doublet with a component corresponding to $\mathrm{V}_{\left(\mathrm{Br}^{-}\right)}$at sites 1 and 2 , and the other one corresponding to $\mathrm{V}_{\left(\mathrm{Br}^{-}\right)}$at sites 3 and 4. The two components merge into a single line for $\mathbf{H} / /[110]$. The essential point is that the anisotropy of the lines $\left|\frac{5}{2}\right| \rightarrow\left|\frac{7}{2}\right|$ nearly vary as $\cos 4 \theta$ which demonstrates a small tilt of the spin Hamiltonian axes away from the crystal axes, and a small contribution of the orthorhombic terms. In other words, the fourth order terms have a dominant contribution and nearly act in the same way as for the $D_{2 d}$ centre. Moreover, for $\theta=(\mathbf{a}, \mathbf{H})=22^{\circ} 30^{\prime}$ the lines of the doublet are separated by about 300 gauss near $T_{1}$ in the tetragonal phase. This large separation prevents line overlap when the lines are shifted and when the line shape is modified (see Fig. $2 b$ ) in the incommensurate phase below $T_{\mathrm{I}}$.

In the following we shall mainly use the $\mathrm{Gd}^{3+}-$ n.n.n. $V_{\left(\mathrm{Br}^{-}\right)}$centre to investigate the incom-

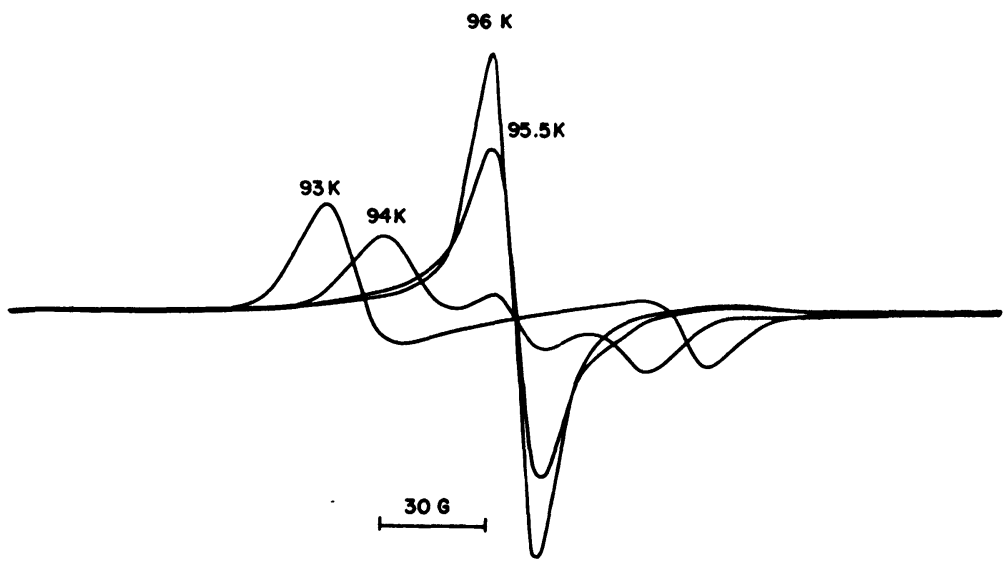

Fig. $2 a .-\theta=22^{\circ} 30^{\prime}$. Line shape above and near $T_{1} \simeq 94 \mathrm{~K}$. On cooling in the range $T_{1}+\varepsilon<96 \mathrm{~K}$ the line becomes markedly Lorentzian. 


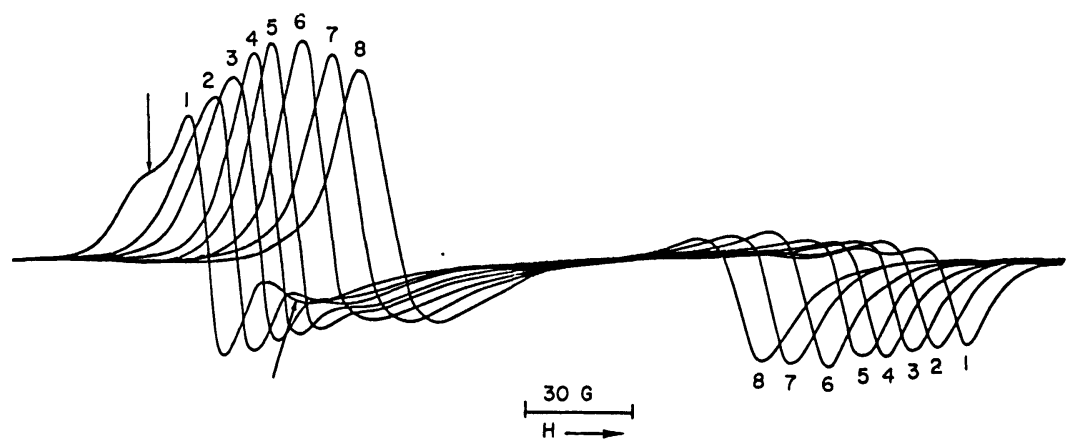

Fig. $2 b .-$ Edge singularities » for $\theta=22^{\circ} 30^{\prime}$.

$$
\begin{array}{llll}
T(1)=79.4 \mathrm{~K} ; & T(2)=84 \mathrm{~K} ; & T(3)=85 \mathrm{~K} ; & T(4)=86 \mathrm{~K} ; \\
T(5)=87 \mathrm{~K} ; & T(6)=90 \mathrm{~K} ; & T(7)=91.8 \mathrm{~K} ; & T(8)=93 \mathrm{~K} .
\end{array}
$$

The arrows denote « Shoulders » which appear on cooling.

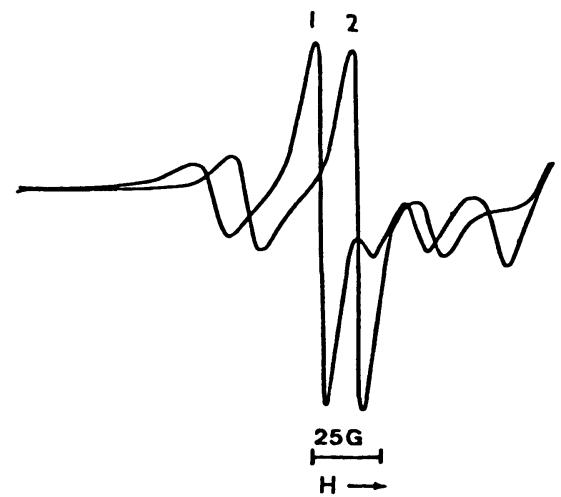

Fig. $2 c$. - Structure of the low field « edge singularity » at low temperature :

$$
T(1)=50 \mathrm{~K} ; T(2)=55 \mathrm{~K} .
$$

The lines of the structure are nearly symmetrical i.e. " commensurate-like ".

mensurate phase : indeed the $\mathrm{D}_{2 \mathrm{~d}}$ and the $\mathrm{Gd}^{3+}-$ n.n. $\mathrm{V}_{\left(\mathrm{Br}^{-}\right)}$lines in the (001) plane exhibit too small an intensity to be easily tractable.

4. Incommensurate phase. - For $\mathbf{H} / / \mathrm{c}$, the lines of both centres, $\mathrm{D}_{2 \mathrm{~d}}$ and $\mathrm{Gd}^{3+}{ }^{+}$.n.n.n. $\mathrm{V}_{\left(\mathrm{Br}^{-}\right)}$, exhibit no significant modification by cooling below $T_{\mathrm{l}}$. A displacement field mainly characterized by a rotation around $\mathrm{c}$ of the local surroundings, of the $\mathrm{Gd}^{3+}$ probe and particularly of the n.n.n. $\mathrm{Br}^{-}$tetrahedron, can account for this result.

For $\mathbf{H}$ in the (001) plane, an important change of the lines $\left|\frac{5}{2}\right| \rightarrow\left|\frac{7}{2}\right|$ of the $\mathrm{Gd}^{3+}$-n.n.n. $\mathrm{V}_{\left(\mathrm{Br}^{-}\right)}$ centre (see Fig. 2 for $\theta=22^{\circ} 30^{\prime}$ and Fig. 3 for $\theta=0^{\circ}$ ) is observed when one undergoes the phase transition. 
$4.1 \theta=(\mathrm{a}, \mathrm{H})=22^{\circ} 30^{\prime}$ (FIGS. 2): - 4.1.1 Pretransitional behaviour (Fig. $\left.2 a\right)$. - When one approaches $T_{1}$ from above, the line exhibits a marked broadening. The line shape, which is nearly Gaussian at room temperature, probably through the unresolved s.h.f. interaction with the $\mathrm{Br}$ nuclei becomes markedly Lorentzian. Rotational fluctuations around $\mathrm{c}$, with increasing amplitude near $T_{1}$, can account for the broadening, and a fast motion regime can account for the Lorentzian component of the line shape.

In a temperature range of a few $0.1 \mathrm{~K}$ around $94 \mathrm{~K}$, the spectrum is characterized by two " edge singularities " accompanied by a central line corresponding to the high-temperature line which vanishes on cooling (Fig. $2 a$ ). A distribution of $T_{\mathrm{I}}$ in the bulk of the sample can trivially account for this observation. Nevertheless, depending on the spectral density $J(\omega, T)$ of the rotational fluctuations, slow frequencies can give rise to a nearly static spectrum with two « edge singularities ", while fast frequencies can give rise to a motional narrowing characterized by a central line. The intrinsic or extrinsic character of this observation is a point worth clarifying.

4.1.2 Incommensurate phase near $T_{1} T_{1}-7<T<T_{1}-\varepsilon$. The high-temperature line is modified into two sharp " edge singularities " separated by a continuous absorption background. Assume a local rotation by $\varepsilon$ of the fourth order around the $x$ axis. From the discussion above, the line is displaced by $\Delta H_{(\varepsilon)} \sim b_{4}^{4} \varepsilon$. This linear dependence accounts for the line shape, in the plane wave modulation regime $\varepsilon(z)=\varepsilon_{\mathrm{A}} \cos \left(k z+\varphi_{0}\right)$. Moreover, the highest sensitivity of the spectra to the phase change consistently occurs for $\theta=22^{\circ} 5$. Small quadratic contributions to the displacement of the line arising from the actual orthorhombic symmetry of the centre can

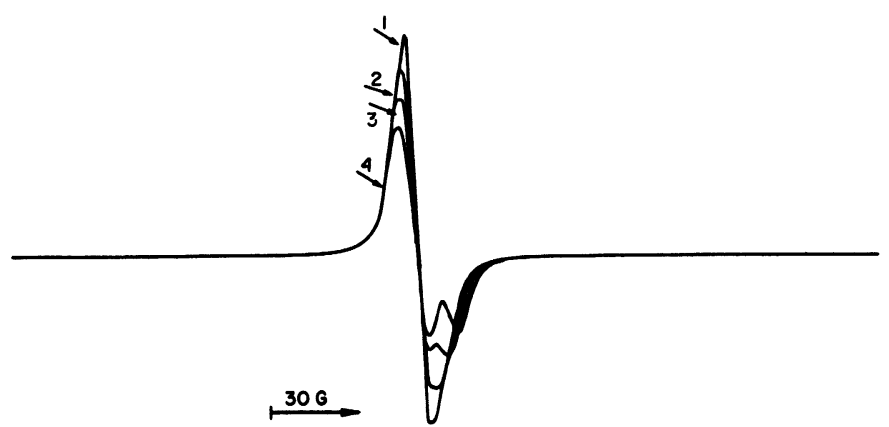

Fig. $3 a$. Line shape for $\theta=0^{\circ}$ near $T_{1}=94 \mathrm{~K}$.

$$
T(1)=95 \mathrm{~K} ; T(2)=94.7 \mathrm{~K} ; T(3)=93.9 \mathrm{~K} ; T(4)=93.5 \mathrm{~K} .
$$

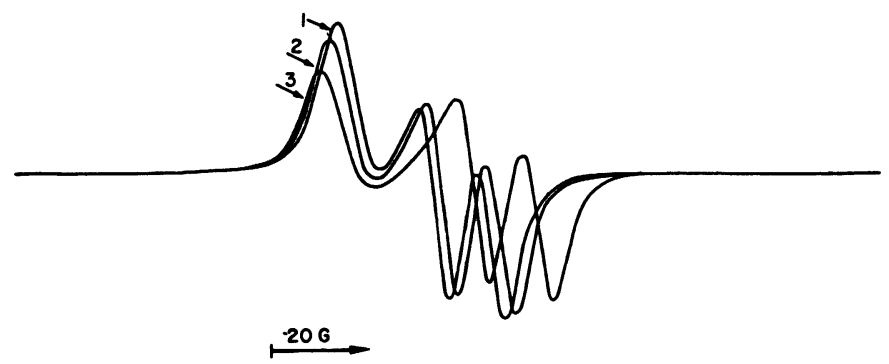

Fig. $3 b .-$ Line shape for $\theta=0^{\circ}$ in the incommensurate phase. Note the three singularities, and $a$ much lower splitting as for $\theta=22^{\circ} 30^{\prime}$.

$$
T(1)=91.2 \mathrm{~K} ; \quad T(2)=90.3 \mathrm{~K} ; \quad T(3)=88.4 \mathrm{~K} ; \quad T(4)=85.8 \mathrm{~K} .
$$


account for the asymmetry of the "edge singularities » (see Fig. 4 in Ref. [1]). Nevertheless we cannot exclude an eventual contribution of broad solitons (see Fig. 7 in Ref. [1]).

The distance between the " edge singularities " permits a study of the temperature dependence of the amplitude $\varepsilon_{\mathrm{A}}$. An exponent law $\varepsilon_{\mathrm{A}} \sim\left(T-T_{\mathrm{I}}\right)^{\beta}$ (Fig. 4) with $\beta=0.34 \pm 0.02$ holds from $T_{1}$ down to $T_{1}-7$. Connected to the high sensitivity of the probe, the question which arises is its ultimate accuracy (through improved temperature regulation) to discriminate between the $(X-Y)$ and Heisenberg values of the critical exponent.

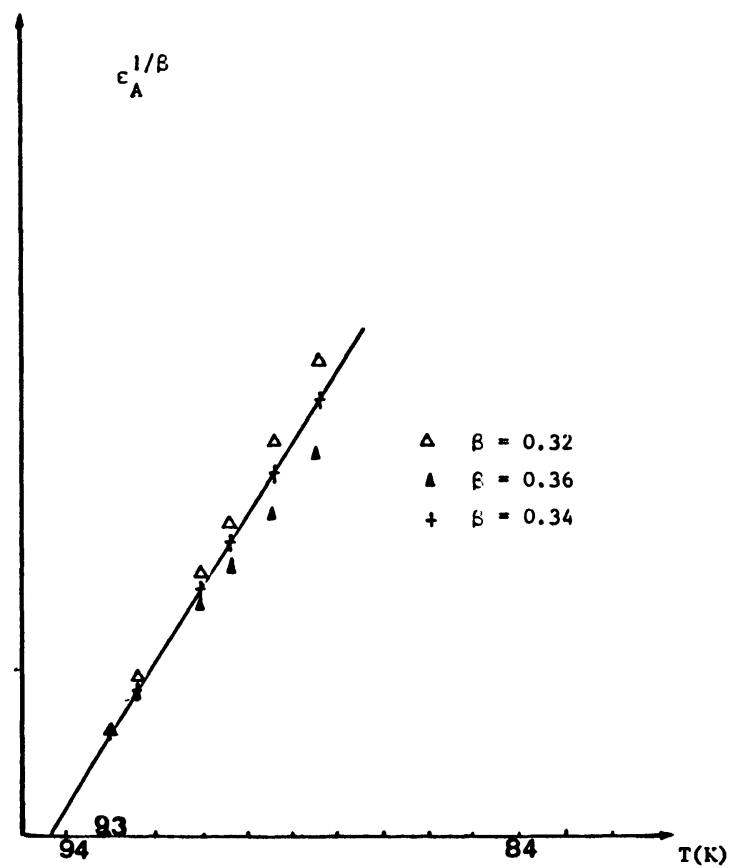

Fig. 4. - Plot of $\varepsilon_{\mathrm{A}}^{1 / \beta}$ versus $T$. $\varepsilon_{\mathrm{A}}$ has been monitored by the distance between the maxima of the absorption spectra, i.e. the zero of the derivative which is actually recorded. The temperature scale is our own temperature scale (see text). The curves for $\beta=0.32$ and $\beta=0.36$ deviate significantly from a straight line.

The absolute value of $\varepsilon_{\mathrm{A}}$ can be directly measured. Indeed, by rotating the crystal by $\pm \varepsilon_{\mathrm{A}}$ around $c$, the " edge singularities » can be observed, one after another, for the same resonant field. We have found $\varepsilon_{\mathrm{A}\left(T_{1}-T=7\right)}=2^{\circ} 10^{\prime}$. From the superposition model this value represents the amplitude of the mean rotation angle of the $\mathrm{Th}-\mathrm{Br}$ bonds.

4.1.3 Low-temperature phase $T<T_{1}-7$ (Fig. 2c). - Below $T=T_{1}-7$, the distance between the absorption maxima deviates from an exponent law and simultaneously a shoulder like structure appears on the low-field « edge singularity » (Fig. 2b). By further cooling (Fig. 2c), this structure develops into " commensurate-like » lines, which would correspond to discrete values of the phase of the modulation. Nevertheless the observed spectra cannot be assigned to a narrow soliton regime [1]. A possibility is a pinning of the phase at particular values by the paramagnetic defect which is used as a probe.

$4.2 \theta=(\mathrm{a}, \mathrm{H})=0$ (FIGS. 3). - According to the analysis above, the line shift should depend on the square of the amplitude of the local displacement field $\varepsilon$ through the fourth order spincrystal field interaction, and the line splitting should be much weaker, as for $\theta=22^{\circ} 30^{\prime}$. Nevertheless, a linear term in $\varepsilon$, can arise from small orthorhombic terms and from the small tilt of the spin Hamiltonian axes. As shown by Blinc et al., the line shape should be characterized by three 
singularities (see Fig. 4 in [1]). This is well verified in figure 3, which is merely shown to illustrate the basic correctness of our straightforward analysis of the E.P.R. spectra.

A more detailed report of this E.P.R. investigation will be given elsewhere.

5. Discussion. - From recent neutron scattering results [7], the $\mathrm{Br}^{-}$displacements below $T_{\mathrm{I}}$ can be described as the superposition of a rotational component and a twist component. Noting $\varepsilon_{\mathrm{R}}\left(\varepsilon_{\mathrm{T}}\right)$ as the amplitude of the rotation (the twist), one gets :

$$
\varepsilon_{\mathrm{R}}=\eta \cos \alpha \cos \phi_{l} \text { and } \varepsilon_{\mathrm{T}}=\eta \sin \alpha \sin \phi_{l} .
$$

$\eta$ represents the amplitude of the modulation which is temperature dependent, and $\phi_{l}$ is the phase of the modulation wave at the crystal site Th $(l)$. From reference [7], we can evaluate the value of $\alpha$ as being about $25^{\circ}$.

A first order expansion of the local line shift is given by :

$$
\Delta H_{l}=R_{\theta} \varepsilon_{\mathrm{R}}+T_{\theta} \varepsilon_{\mathrm{T}}+0\left(\varepsilon_{\mathrm{R}}^{2}, \varepsilon_{\mathrm{T}}^{2}, \varepsilon_{\mathrm{R}} \varepsilon_{\mathrm{T}}\right) .
$$

The parameters $R_{\theta}, T_{\theta}$ depend on the orientation $(\theta)$ of the magnetic field in the [001] plane and characterize the individual contribution of the rotation (twist) to the line shift.

Let us assume $R_{\theta} \gg T_{\theta}$, i.e. a line shift much more sensitive to the rotation than to the twist. Then we obtain consistency between neutron measurements and E.P.R. results. This assumption is well supported by the high value of $b_{4}^{4}$ which is amplified by the factor of 4 which appears in the angular dependence of $R_{\theta} \propto \mathrm{d} \cos 4 \theta / \mathrm{d} \theta=-4 \sin 4 \theta$. Moreover the amplitude of $\varepsilon_{\mathrm{R}}$ is larger by a factor 2 than the amplitude of $\varepsilon_{\mathrm{T}}(\cos \alpha / \sin \alpha \simeq 2)$. Note that our measurement of the amplitude $\varepsilon_{\mathrm{R}}=3.5 \times 10^{-2}$ radian is in agreement with the value of $\eta$ measured by neutron scattering (a few percent in units of $a$ ). Note also that, neglecting any phase pinning effects, the " edge singularities " at $\theta=22^{\circ} 30^{\prime}$ would correspond to a nearly pure rotation of the $\mathrm{ThBr}_{4}$ groups i.e. $\phi_{l} \simeq 0, \pi$.

Of course a more detailed investigation would be necessary to analyse the symmetry lowering effects which arise through the twist of the $\mathrm{Br}^{-}$anions and through the n.n.n. vacancy and the structure of the « edge singularities » which develop at low temperature.

\section{References}

[1] Blinc, R., Aleksandrova, I. P., Chavec, A. S., Mila, F., Rutar, R., Seliger, J., Popic, B. and Zumer, S., J. Phys. C : Solid State Phys. 15 (1982) 547-563.

[2] Pezeril, M., Emery, J. et Fayet, J. C., J. Physique-Lett. 41 (1980) L-499-L-502.

[3] Pezeril, M. et Fayet, J. C., J. Physique-Lett. 43 (1982) L-267-L-272.

[4] Hubert, S., Delamoye, P., Lefrant, S., Lepostollec, M. and Hussonnois, M., J. Solid State Chem. (1981) 36-43.

[5] Khan, M., Peneau, A., Guibe, L., Delamoye, P. et Hussonnois, M., J. Molec. Struct. (1982), in press.

[6] Currat, R., J. Physique Colloq. 42 (1981) C6-693-C6-700.

[7] Bernard, L., Currat, R., Delamoye, P., Zeyen, C., Hubert, S. and De Kouchkovski, R., accepted in $J$. Phys.

[8] De Kouchkovski, R., Le Cloarec, M. F. and Delamoye, P., Mat. Res. Bull. 16 (1981) 142.

[9] Fayet-Bonnel, M., Buzare, J. Y. and Fayet, J. C., Solid State Commun. 38 (1981) 37-38.

[10] Hussonnois, M., Krupa, J. C., Genet, M., Brillard, L. and Carlier, R., J. Cryst. Growth 51 (1981) 11-16.

[11] Hubert, S., Edelstein, N. and Emery, J., to be published.

[12] Newman, D. J., Urban, W., Adv. Phys. 24 (1975) 793-844. 